\title{
PRUEBAS IN VITRO DE HONGOS ANTAGONISTAS PARA COMBATIR LA ROYA DE MORA EN COSTA RICA ${ }^{1}$
}

\author{
Konrad Holst Sanjuán ${ }^{* *}$, German Rivera-Coto ${ }^{* *}$, Keylor Villalobos-Moya*, Rafael Orozco- \\ Rodríguez ${ }^{2 / *}$, Steffany Orozco-Cayasso**
}

Palabras clave: Rubus sp.; mora; biocontrol; parasitismo; roya; pruebas in vitro.
Keywords: Rubus sp.; blackberry; bio control; parasitism; rust; in vitro tests.

Recibido: 23/08/17

\section{RESUMEN}

El objetivo de esta investigación fue aislar hongos asociados a pústulas de roya (Kuehneola spp.), para seleccionar cepas con algún efecto antagonista sobre el agente causal de esta enfermedad, en 7 localidades de la Zona de Los Santos y Pérez Zeledón, Costa Rica. Se realizaron muestreos en plantaciones de mora cultivadas orgánicamente para obtener distintos hongos y determinar su posible acción antagónica contra el agente causal. El estudio se extendió por espacio de un año (junio 2011-junio 2012), periodo en el cual se realizaron recolecciones mensuales para incluir la mayor diversidad de eventuales biocontroladores durante un ciclo. Del material recolectado se logró aislar un total de 11 géneros diferentes de hongos: Pestalotia spp., Sphaeropsis sp., Botrytis sp., Verticillium sp., Macrophomina sp., Cladosporium spp., Phoma sp., Colletotrichum spp., Rhizoctonia sp., Libertella sp., Paecilomyces sp., y varios micelios estériles no identificados. Para establecer el posible efecto antagonista de los hongos, se realizaron ensayos in vitro con el fin de determinar su capacidad supresora, sobre la germinación de las uredósporas de Kuehneola

1 Este trabajo fue financiado por un proyecto FEES 2009 "Generación de conocimiento científico y tecnológico sobre la biodiversidad de moras criollas costarricenses con alto potencial antioxidante".
Aceptado: 18/01/17 
spp. De todos los aislamientos, los que resultaron significativamente distintos a su respectivo testigo fueron los del género Cladosporium. Para determinar diferencias significativas entre estos aislamientos se realizaron comparaciones entre ellos y se estableció que todos los aislamientos afectaron de igual forma la germinación de uredósporas y solamente Clados4 inhibió por completo la germinación.

\section{INTRODUCCIÓN}

En Costa Rica el cultivo de la mora (Rubus spp.) es desarrollado por pequeños productores, que en su mayoría la cultivan como una opción para diversificar su actividad agrícola. Los principales lugares donde se ha desarrollado este cultivo son la zona de Los Santos, El Guarco y Pérez Zeledón. Según datos de Castro y Cerdas (2005) esta actividad proporciona un ingreso económico importante para los agricultores y de ella dependen alrededor de 800 familias.

Industrialmente es un fruto de alto valor, por la cantidad de productos que se pueden elaborar a partir de él, además de la comercialización convencional como fruta fresca (Castro y Cerdas 2005). A estas opciones, debe sumársele la posibilidad de producirla de forma orgánica, lo cual ha abierto un nicho de mercado para exportación y competencia con otros países que producen a un menor costo, pero de forma convencional. Por esta razón, hay interés en desarrollar técnicas de cultivo que apoyen la producción orgánica y preserven esta modalidad de agricultura, entre los productores de mora.

Uno de los problemas para la producción y exportación de frutos, lo constituye la roya, la cual afecta el follaje y reduce la calidad de los frutos. Aparte de los efectos nocivos sobre la producción, las lesiones en las drupas ocasionan pérdidas económicas por el rechazo en las plantas their suppressive capacity on Kuehneola spp., uredospores germination. Of all the isolates, those that were significantly different from their respective control were those of Cladosporium spp. In order to determine significant differences between these isolates, comparisons were made between them, and it was established that all the isolates equally affected germination of uredospores and only Clados4 completely inhibited germination.

empacadoras. El agente causal de esta enfermedad es Kuehneola spp., patógeno que se encuentra ampliamente distribuido en Centro América y algunos países de América del Sur. En todas esas regiones se informa de una amplia variedad de especies del género Rubus, como hospedantes de esta enfermedad (Yun 2010).

Una alternativa viable pero poco estudiada, para enfrentar el problema señalado, es el combate de esta enfermedad con agentes biológicos. Para lograrlo de forma eficiente, es necesario conocer e identificar primero los organismos autóctonos de las zonas de producción, que tienen el potencial de actuar como controladores. De esta manera, se pretende apoyar a los productores con tecnologías que les ayuden a manejar mejor sistemas de producción, amigables con el ambiente y que permitan mantener la característica orgánica, como valor agregado a su producto.

Para estos procesos se prefiere utilizar antagonistas nativos debido a que tienen un mayor potencial para combatir una enfermedad. Esto se debe principalmente a que se encuentran adaptados al mismo hábitat del cultivo (Roiger et al. 1991, Andrews 1992, Michel et al. 2001). La presente investigación pretendió contribuir al proceso señalado, para lo cual se realizó la recolección de hongos antagonistas y se hizo una selección inicial mediante pruebas in vitro contra Kuehneola spp. 


\section{MATERIALES Y MÉTODOS}

La toma de muestras para este estudio se realizó en plantaciones de mora orgánica ubicadas en 7 localidades de la zona de Los Santos y Pérez Zeledón, Costa Rica (Cuadro 1). Estas comunidades fueron: San Martin y Cedral de León Cortez, Bajo Canet de Tarrazú, La Luchita del Guarco, Buena Vista, Jardín y División de Pérez Zeledón.

Cuadro 1. Ubicación e información de las plantaciones de mora de donde se obtuvieron las muestras para el estudio, Costa Rica, 2011.

\begin{tabular}{|c|c|c|c|c|}
\hline Productor & Localidad & Cantón & Ubicación & Altitud (msnm) \\
\hline Miguel Mena & La Luchita & El Guarco & $\begin{array}{l}\text { N 0944'39.9"' } \\
\text { W083'56'15.7" }\end{array}$ & 1935 \\
\hline Didier Jiménez & San Martín & León Cortés & $\begin{array}{l}\text { N 0943'43.2", } \\
\text { W084'00'06.3", }\end{array}$ & 1864 \\
\hline Plantas silvestres & Cedral & León Cortés & $\begin{array}{l}\text { N } 09^{\circ} 42^{\prime} 50.9^{\prime}, \\
\text { W083'58'19.2" }\end{array}$ & 2218 \\
\hline Edwin Martínez & Bajo Canet & Tarrazú & $\begin{array}{l}\text { N 0942'01.3"' } \\
\text { W083'59'49.0" }\end{array}$ & 1840 \\
\hline Franco Portugués & Buena Vista & Pérez Zeledón & $\begin{array}{l}\text { N 09³0'15.3", } \\
\text { W083'39'28.4", }\end{array}$ & 1709 \\
\hline Rafael Céspedes Zúñiga & División & Pérez Zeledón & $\begin{array}{l}\text { N 09³0'27.6", } \\
\text { W08341'31.4" }\end{array}$ & 1958 \\
\hline Raúl Araya Atencio & División & Pérez Zeledón & $\begin{array}{l}\text { N 09³0’35.1" } \\
\text { W08340'39.9”, }\end{array}$ & 1869 \\
\hline José Gerardo Gamboa & Jardín & Pérez Zeledón & $\begin{array}{l}\text { N 09³0'08.3', } \\
\text { W083'41'29.4" }\end{array}$ & 1916 \\
\hline
\end{tabular}

El periodo de recolección se extendió por un año (jun. 2011-jun. 2012), con recolecciones mensuales para incluir las variaciones estacionales de la roya y sus eventuales biocontroladores. El trabajo de laboratorio consistió en el aislamiento y pruebas in vitro de los hongos asociados a pústulas de roya y se realizó en el Laboratorio de Fitopatología de la Universidad Nacional de Costa Rica (UNA). En cada localidad se recolectaron muestras de hojas y frutos con síntomas de roya. Para el caso particular de las hojas con síntomas de roya, se separaron los foliolos según su apariencia (Figura 1) en las siguientes categorías:

$(\mathrm{SH})=$ Foliolos donde las pústulas no tienen una producción activa de uredósporas.

(B) $=$ Foliolos con pústulas activas y crecimiento blanquecino sobre las uredósporas.

$(\mathrm{H})=$ Foliolos con pústulas activas de tamaño reducido.

$(\mathrm{N})=$ Foliolos con pústulas inactivas y un punto necrótico en la parte central.

$(\mathrm{F})=$ Frutos con pústulas. 


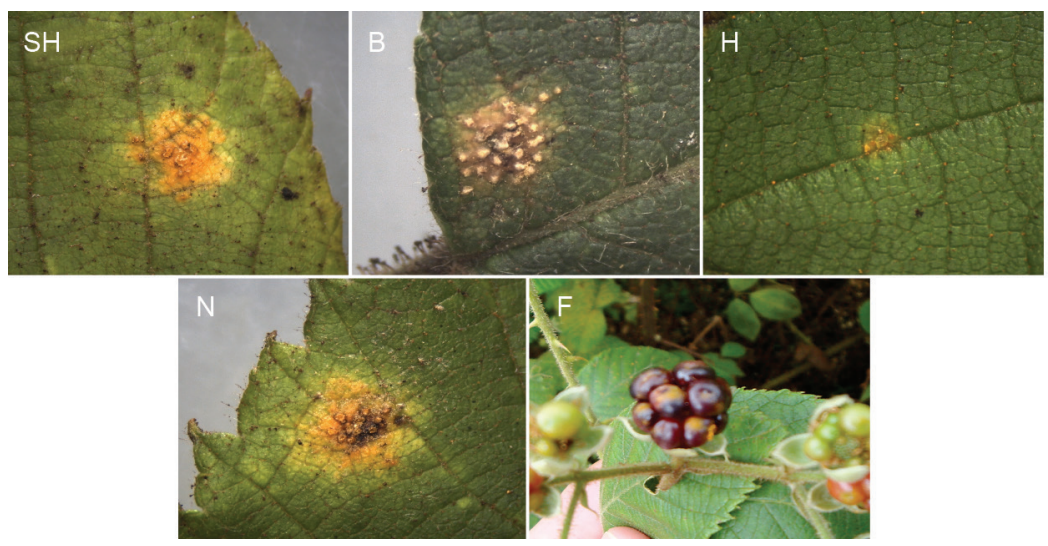

Fig. 1. Categorías de pústulas de Kuehneola spp., en hojas y frutos clasificadas según su apariencia.

$(\mathrm{SH})=$ foliolos donde las pústulas no tienen una producción activa de uredósporas.

(B)=foliolos con pústulas activas y crecimiento blanquecino sobre las uredósporas.

$(\mathrm{H})=$ foliolos con pústulas activas de tamaño reducido.

$(\mathrm{N})=$ foliolos con pústulas inactivas y un punto necrótico en la parte central.

$(\mathrm{F})=$ frutos con pústulas.

\section{Aislamiento de los posibles organismos antagonistas}

El procedimiento consistió en colocar sobre una pieza de cerámica previamente flameada el foliolo enfermo y con ayuda de un bisturí estéril se cortó la pústula completa. Las secciones, así obtenidas, se colocaron sobre una gasa estéril, hasta formar grupos de 25 aproximadamente, luego la gasa se dobló y selló con cinta adhesiva, para proceder a la desinfección superficial, dentro de una cámara de flujo laminar. Esta se realizó de la siguiente forma: primero se sumergió la gasa con las pústulas, en un recipiente con alcohol etílico al $75 \%$ por 30 segundos. Seguidamente se extrajo y se transfirió (usando pinzas estériles) a otro recipiente con hipoclorito de sodio $(\mathrm{NaOCl})$ al $0,5 \%$ por 60 segundos. Finalmente, se realizaron 2 lavados consecutivos con agua destilada estéril.

En la cámara se utilizaron platos Petri con el medio papa-dextrosa-agar (PDA) acidificado (2 gotas de ácido láctico al $25 \%$ por plato) y sobre él se colocaron 4 pústulas desinfectadas, en forma equidistante.
Para el procesamiento de las muestras clasificadas en la categoría B se utilizó un método de aislamiento directo. Para esto se colocaron las muestras en refrigeración por 24 horas para inhibir el crecimiento de ácaros. Luego se transfirieron los foliolos a la cámara de flujo laminar dentro de una placa Petri, y mediante una jeringa con agua destilada estéril, se colocó una gota sobre la pústula. Seguidamente, con una asa bacteriológica estéril se agitó el líquido sobre la pústula y se realizó un estriado en la superficie del medio PDA acidificado dentro de platos Petri. Cuando el material correspondió a frutos, estos se fragmentaron al separar las drupas enfermas con ayuda de pinzas estériles. Las drupas extraídas se colocaron sobre piezas de gasa estéril para desinfectarlas y cultivarlas de la forma descrita para las pústulas provenientes de foliolos.

\section{Recolección de uredósporas para las pruebas de antagonismo}

Previo a las pruebas de antagonismo, se recolectaron en el campo hojas de mora con roya en plena esporulación para cosechar la mayor 
cantidad posible de uredósporas. Esto se hizo con un pincel para barrer las esporas presentes sobre las pústulas. El barrido se realizó sobre un papel encerado, con cuidado de que en este cayera todo el material desprendido, para luego recogerlo y envolverlo en el mismo papel. Este se rotuló y se colocó dentro de un vial para almacenarlo a $10^{\circ} \mathrm{C}$ hasta su uso.

Las hojas barridas se conservaron para volver a cosechar más uredósporas. Para lograrlo se insertaron los pecíolos en una espuma floral (oasis) humedecida, dentro de una caja plástica mantenida en una incubadora a $15^{\circ} \mathrm{C}$. Esto permitió conservar el material y poder realizar de 2 a 3 recolecciones a partir de un mismo material colectado en el campo.

\section{Preparación de las suspensiones de esporas para las pruebas de antagonismo}

La suspensión de conidios del hongo a probar, se preparó con una concentración de $1 \times 10^{6}$ conidios por mililitro. Para esto se tomó un plato con cultivo puro y esporulado del antagonista a evaluar, se raspó el micelio con una espátula metálica estéril y se colocó el raspado en un Beaker de $100 \mathrm{ml}$ que contenía $20 \mathrm{ml}$ de agua destilada estéril, a la cual se le agregó, Tween 20 como dispersante y cefalexina $\left(2,5 \mathrm{mg} \cdot \mathrm{ml}^{-1}\right)$ para evitar el crecimiento de bacterias.

Con las uredósporas de Kuehneola spp., se preparó una suspensión en agua destilada estéril más Tween 20 y cefalexina a una concentración de $2 \times 10^{4}$ uredosporas por mililitro, concentración utilizada por Johnson y Mahaffe (2010), en ensayos similares. Con el fin de remover cualquier inhibidor natural de germinación, se realizó un lavado de las uredósporas, suspendiéndolas primero en agua estéril y luego filtrándolas al vacío. De las esporas recuperadas se preparó la suspensión respectiva. En todos los casos las concentraciones de esporas por mililitro, se determinaron con la ayuda de un hematocímetro, según la metodología propuesta por French y Hebert (1980).

\section{Ensayos in vitro para seleccionar los hongos con efecto antagonista}

Las pruebas fueron hechas en portaobjetos estériles con una fina capa solidificada del medio agar-agua (AA) en la cara superior, sobre ella se depositó una alícuota de $25 \mu 1$ de la suspensión de conidios del hongo a probar y se esparció con una barra de vidrio sobre el medio. Una hora después, se colocaron y esparcieron $25 \mu 1$ de la suspensión uredósporas previamente preparada y se esparció de igual forma. Las láminas se colocaron dentro de una cámara húmeda, conformada por una placa Petri con papel absorbente al fondo, humedecido con agua destilada. Como soporte para el portaobjetos se utilizaron 2 mondadientes, todos estos materiales fueron previamente autoclavados.

Para cada hongo se realizaron 5 repeticiones y 24 horas después se valoraron. En las evaluaciones se contaron 20 uredósporas al azar por cada repetición, a lo largo de toda la lámina, contabilizándose las uredósporas germinadas y no germinadas. Se consideró como germinada aquella que desarrolló un tubo germinativo igual o mayor al diámetro de la espora.

\section{Diseño experimental}

Se utilizó un diseño completamente al azar con 5 repeticiones por tratamiento para cada una de las 6 evaluaciones realizadas. En cada evaluación, se probaron de 2 a 3 aislamientos de hongos y un testigo. La unidad experimental consistió en un portaobjetos colocado en cámara húmeda dentro de una placa Petri. El portaobjetos tenía una capa fina del medio AA en su cara superior y en el lado inferior, se hicieron 2 líneas con marcador permanente que delimitaban un área de $2,5 \mathrm{~cm}^{2}$. En el centro del área delimitada, se colocó la alícuota de $25 \mu \mathrm{l}$ de la suspensión de uredósporas y la de $25 \mu \mathrm{l}$ con esporas del hongo a evaluar. Para el testigo se colocaron $25 \mu \mathrm{l}$ de agua destilada estéril en lugar del hongo. Cada hongo evaluado representaba un tratamiento y se hizo 5 repeticiones por tratamiento. 


\section{Análisis estadístico}

Debido a la naturaleza binomial de los datos (uredósporas germinadas o no germinadas) y a la realización de diferentes evaluaciones en el tiempo, se procedió primero a comparar los tratamientos dentro de cada evaluación mediante una regresión logística. Este análisis se realizó con el procedimiento GenMod de SAS 9,2.

Se procedió como primer paso, a obtener la diferencia de cada hongo en cada evaluación con respecto a su testigo. En un segundo paso, al resultado obtenido se le restó la diferencia del hongo de la otra evaluación, y se obtuvo el error estándar de dicha diferencia. Con esta información se calculó un valor de Chi cuadrado y la probabilidad respectiva que indicó si existe o no diferencia entre el par de hongos de diferentes evaluaciones.

\section{RESULTADOS Y DISCUSIÓN}

\section{Aislamiento de los posibles organismos antagonistas}

Dentro del periodo de estudio, se lograron aislar de las pústulas de Kuehneola spp., un total de 11 géneros diferentes de hongos (Cuadro 2). Estos organismos se encontraban asociados a las pústulas en una comunidad multigenérica donde cada participante interactuó con el agente etiológico de formas diversas. Entre la población obtenida había una importante cantidad de hongos saprófitos, patógenos de la mora, posibles parásitos de Kuehneola spp., así como parásitos que no tienen relación con esta enfermedad, pero que pueden competir en la colonización de los tejidos del hospedero (Moricca et al. 2001).

Cuadro 2. Lista de géneros y cantidad de aislamientos fungosos, obtenida de pústulas de Kuehneola spp., por género en cada localidad muestreada, junio 2011-junio 2012.

\begin{tabular}{lcccccccc}
\hline & La Luchita & San Martin & Bajo Canet & Buena Vista & División & Jardín & Cedral & Total \\
\hline Botrytis & 2 & 0 & 0 & 0 & 0 & 0 & 0 & 2 \\
Cladosporium & 2 & 2 & 5 & 0 & 2 & 1 & 1 & 13 \\
Colletotrichum & 0 & 1 & 3 & 0 & 0 & 0 & 0 & 4 \\
Libertella & 1 & 0 & 0 & 0 & 0 & 0 & 0 & 1 \\
Macrophomina & 3 & 0 & 0 & 0 & 0 & 0 & 0 & 3 \\
N.I.P.* & 0 & 0 & 0 & 0 & 0 & 0 & 1 & 1 \\
Paecilomyces & 0 & 0 & 0 & 0 & 0 & 1 & 0 & 1 \\
Pestalotia & 3 & 2 & 5 & 5 & 1 & 4 & 2 & 22 \\
Phoma & 0 & 0 & 0 & 0 & 0 & 0 & 1 & 1 \\
Rhizoctonia & 0 & 1 & 0 & 0 & 0 & 1 & 0 & 2 \\
Sphaeropsis & 3 & 0 & 0 & 0 & 0 & 0 & 0 & 3 \\
Verticillium & 1 & 0 & 0 & 0 & 0 & 0 & 0 & 1 \\
\hline Total & 15 & 6 & 13 & 5 & 3 & 7 & 5 & 54 \\
\hline
\end{tabular}

*N.I.P, Hongo no identificado con picnidios. 
Los organismos aislados tenían la habilidad de habitar en las pústulas producidas por Kuehneola spp., y son representantes de una muestra de la microflora fungosa endémica en las plantaciones de mora de esa región del país. (Chaverri 2012. Especies silvestres de Trichoderma con base en su historia natural y evolutiva para el control biológico de enfermedades de árboles tropicales (correo electrónico). Maryland, Estados Unidos, UMD (University of Maryland)) resalta la importancia de buscar agentes de control biológico en plantas dentro de su hábitat natural, y centros de origen, debido a que ellos se encuentran aclimatados a las zonas de producción y pueden llegar a ejercer su acción antagónica eficientemente.

Se pudieron identificar dentro de los aislamientos algunos patógenos de importancia en el cultivo de la mora como lo son Botrytis sp., y Colletotrichum spp. (Castro y Cerdas 2005, Orozco 2003). Pero además se aisló un grupo de hongos constituido por varios aislamientos de los géneros: Cladosporium spp. Verticillium sp., y Paecilomyces sp., los cuales se citan en la literatura como controladores biológicos y algunos como potenciales antagonistas de las royas (Canjura et al. 2002, Moricca et al. 2001, Tsuneda y Hiratsuka 1979).

Una clara tendencia observada en los datos del Cuadro 2, es que los hongos predominantes durante el periodo de estudio, fueron Pestalotia spp., y Cladosporium spp., géneros que estuvieron presentes en todos los puntos de muestreo, a excepción del género Cladosporium que no se obtuvo en Buena Vista.

En la Figura 2 se observan los géneros aislados según el tipo de pústula donde crecían originalmente. En el gráfico destaca Pestalotia

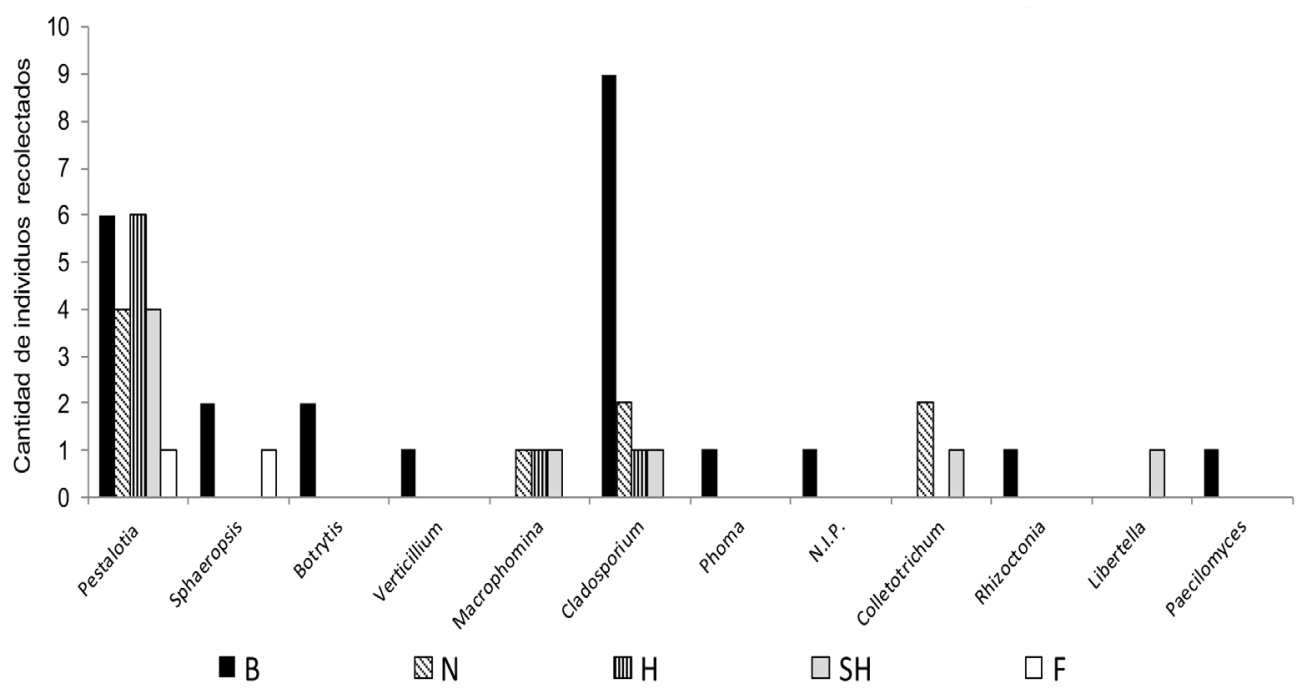

Fig. 2. Cantidad de géneros obtenidos de aislamientos realizados en los distintos tipos de pústulas.

(B)=pústulas activas y con crecimiento blanquecino.

$(\mathrm{N})=$ pústulas inactivas con un punto necrótico en la parte central.

$(\mathrm{H})=$ pústulas activas de tamaño reducido.

$(\mathrm{SH})=$ pústulas sin producción activa de uredósporas.

(F)=pústulas de frutos, junio 2011-junio 2012. 
spp., como el hongo más frecuente en todos los tipos de pústula. Esto puede deberse a que es un hongo comúnmente aislado en los foliolos de las plantas, como un habitante del filoplano. También este género es considerado como un patógeno débil que en forma oportunista se establece en lesiones previas causadas por otros organismos, por lo tanto, al estar las pústulas abiertas encuentra un sitio propicio para establecerse (Mouen et al. 2008, Espinoza y Briceño 2008, Agrios 2005 y Arauz 2011).

El otro género más frecuente fue Cladosporium spp., el cual fue recuperado a partir de la mayoría de los tipos de pústula. Sin embargo, destaca la presencia mayoritaria en las pústulas con crecimiento blanquecino. Es importante señalar, que en la población aislada a partir de este tipo de pústula, también se obtuvo otro hongo que la literatura señala como antagonista de las royas y corresponde al género Verticillium sp., algunas veces citado en la literatura como Lecanicillium sp. (Canjura et al. 2002).
El crecimiento blanquecino, que se observaba en las pústulas de Kuehneola spp., probablemente es causado por el crecimiento micelial de alguno de los hongos aislados, que se nutren de las uredósporas. Tsuneda y Hiratsuka (1979) informan haber observado agallas de la roya Endocronartium harknessii cubiertas por hongos secundarios que aparentemente destruían la superficie de los soros de la roya. Esta habilidad de interactuar con el patógeno es una de las características más importantes que se buscan en un buen biocontrolador (Chaverri 2012).

\section{Comparación estacional de los aislamientos y pruebas in vitro}

En la Figura 3 se puede apreciar las frecuencias con las cuales se presentó cada uno de los aislamientos en la estación lluviosa y la seca. Al compararlos, se observa que en la época lluviosa se logró aislar más individuos que en la época seca. Sin embargo la diversidad de géneros

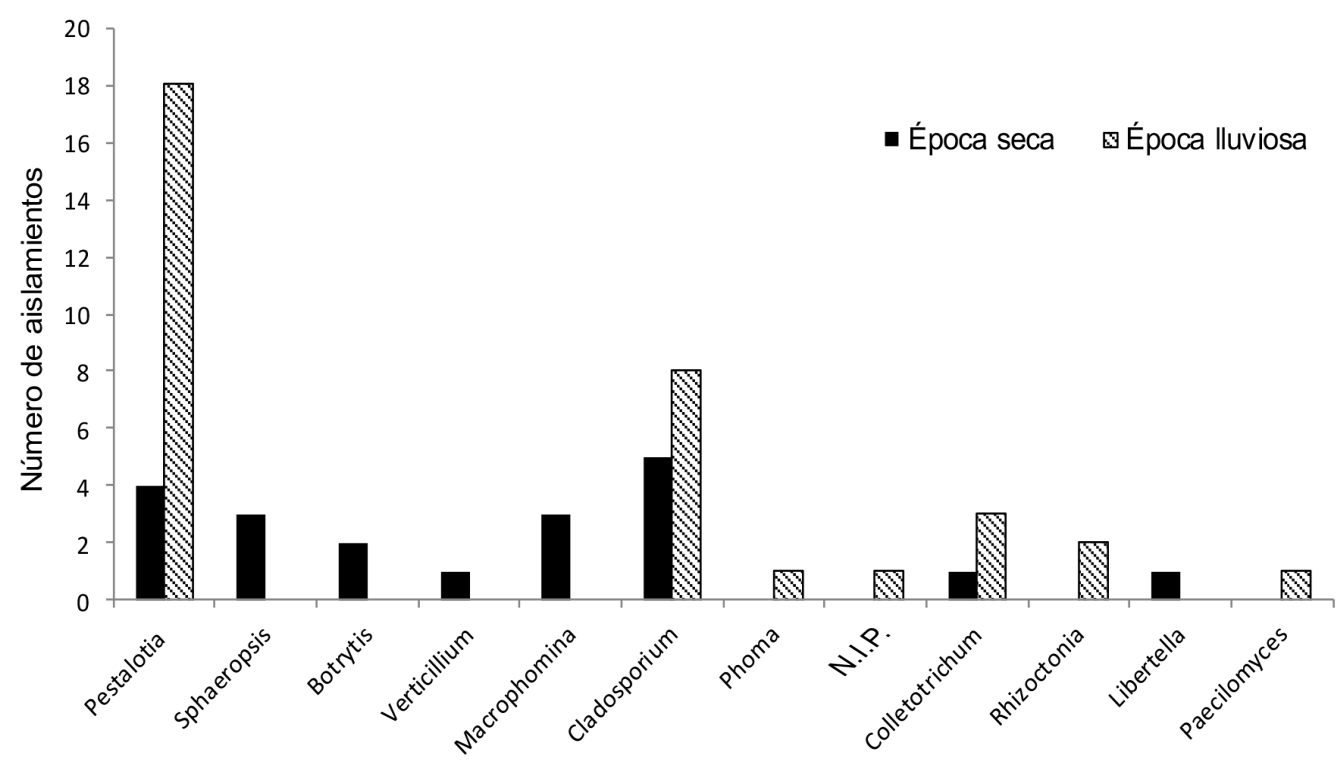

Fig. 3. Total de aislamientos obtenidos a partir de pústulas de Kuehneola spp., tanto en la época seca como lluviosa, junio 2011-junio 2012. 
recolectados fue similar entre ambos periodos pues en la época lluviosa se aislaron 7 géneros distintos, mientras que en la seca se llegó a 8. El mayor número de aislamientos observados en la época lluviosa correspondió a Pestalotia spp. Esto se explica por las características del hongo que se comporta como un oportunista y patógeno secundario, favorecido por las condiciones prevalecientes en la estación lluviosa (Mouen et al. 2008, Espinoza y Briceño 2008).

Para las pruebas in vitro se usaron 11 aislamientos de Cladosporium spp. (Clados), uno de Paecilomyces sp. (Paecilo), uno de Pestalotia sp. (Pest), uno de Sphaeropsis sp. (Sphae), uno de Libertella sp. (Liber) y uno de Phoma sp. (Phom). Los resultados se resumen en la Figura 4 (a - f). Por lo minucioso de este trabajo y la cantidad de observaciones bajo el microscopio hubo necesidad de hacer las pruebas en distintos momentos con el fin de poder probar los géneros aislados. De estas evaluaciones se dejaron de lado algunos aislamientos, ya sea porque eran fitopatógenos reconocidos o porque no fue posible hacerlos producir esporas, este es el caso de Verticillum sp., que después de varios intentos, con el uso de distintos medios y técnicas de cultivo, no esporuló in vitro. 

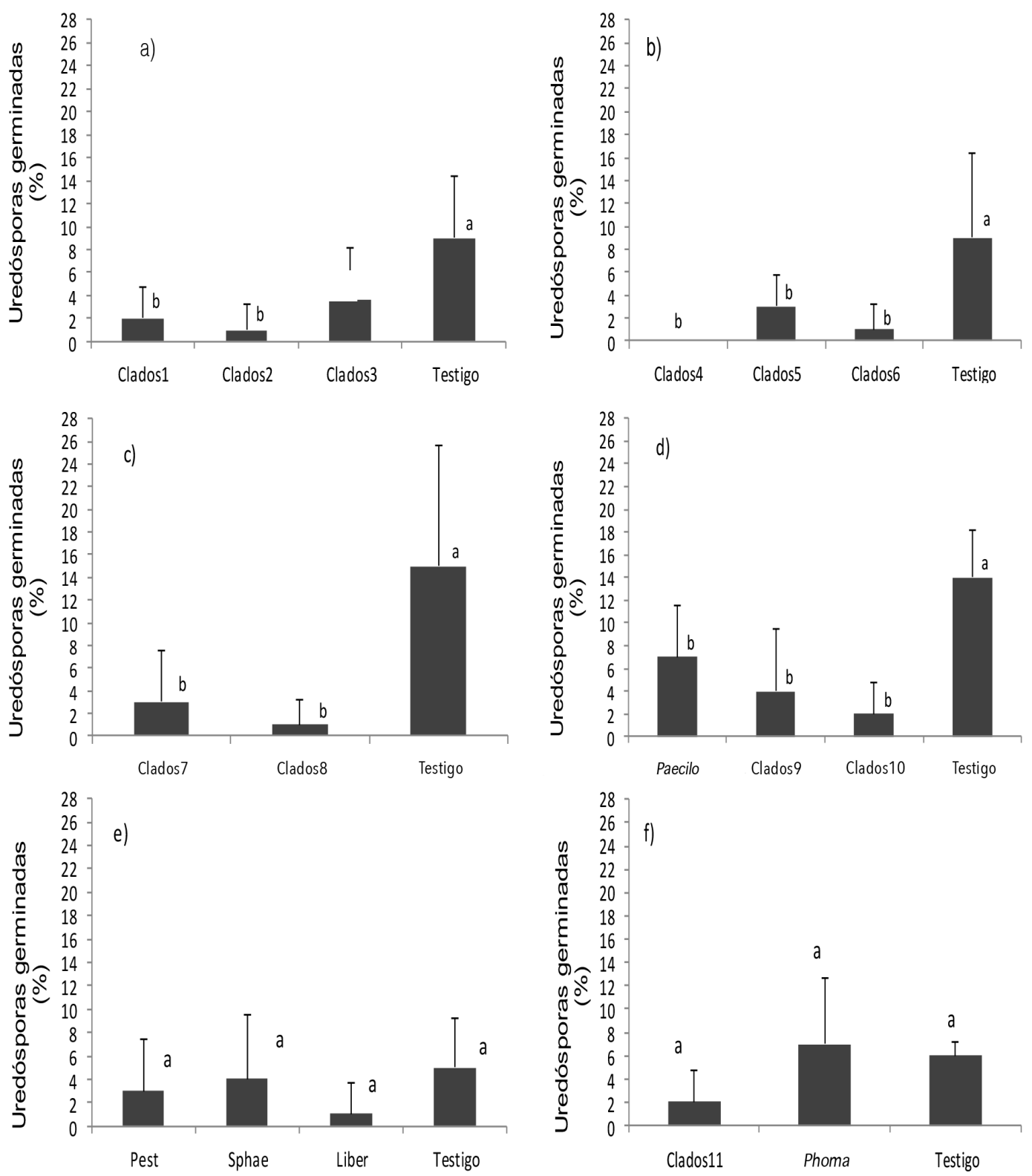

*Letras iguales dentro de la misma figura significa que no difieren estadísticamente.

Fig. 4. Porcentaje de germinación de uredósporas de Kuehneola spp., en interacción con distintos hongos evaluados para determinar posibles antagonismos. 
La Figura 4a) representa los resultados de la primera prueba donde se observa que los aislamientos clados1 y clados 2 fueron significativamente distintos a su respectivo testigo $(\mathrm{p}=0,0239)$ que mantuvieron los porcentajes de germinación de las uredósporas por debajo del 2\%. En la segunda prueba (Figura 4b) se observa una disminución significativa $(\mathrm{p}=0,0151)$ en la germinación de uredósporas con los aislamientos Clados4 y Clados6 respecto al testigo. De ellos destaca el aislamiento Clados4, que mantuvo consistente un valor de $0 \%$ de germinación en todas las repeticiones. Este resultado es probable que se deba a la acción combinada de varios mecanismos responsables del antagonismo contra Kuehneola spp., que no permite ni siquiera la germinación de las uredósporas.

En la tercera evaluación (Figura 4c) los aislamientos Clados7 y 8 produjeron una disminución significativa en la germinación de las uredósporas $(\mathrm{p}=0,0001)$, al igual que los aislamientos Clados9 y 10 (Figura 4d) de la cuarta valoración $(\mathrm{p}=0,0051)$. En esta última evaluación el tratamiento Paecilo no logró disminuir la germinación a un nivel que se pudiera considerar como significativamente distinta a su testigo. En la quinta y sexta estimación (Figura 4e y 4f) no se obtuvo diferencias significativas entre tratamientos ( $\mathrm{p}=0,3587$ y $\mathrm{p}=0,1845$ respectivamente). Esto pudo ser debido a que los hongos probados en su mayoría no son géneros conocidos como antagonistas.

En la Figura 4 se observa que de todos los aislamientos evaluados solo resultaron significativamente distintos a su testigo los de Cladosporium spp. (Clados1, 2, 4, 6, 7, 8, 9 y 10). Este resultado coincide con lo que la literatura señala para el género Cladosporium, considerado en varios trabajos como hiperparásito agresivo de royas, en las cuales coloniza los distintos tipos de esporas producidos por ellas (Sharma y Heather 1978, Moricca et al. 2001). Resultados de Moricca et al. (2001), indican que C. tenuissimum inhibe el crecimiento y desarrollo de una de las roya del pino (Cronatium flaccidum), en ensayos realizados bajo condición de invernadero.
Puede agregarse además que los aislamientos de Cladosporium spp., que resultaron significativamente distintos a sus respectivos testigos, provienen de recolecciones realizadas tanto de la época seca como lluviosa. Por la frecuencia observada y la persistencia estacional, puede afirmarse que es un hongo endémico y activo durante todo el año; características deseables en un controlador biológico, bien adaptado a las condiciones ecológicas de la zona (Chaverri 2012).

\section{Comparación entre aislamientos de Cladosporium spp.}

Con el fin de lograr una mejor visión del efecto antagonista entre los aislamientos que redujeron significativamente la germinación de uredósporas, se realizaron comparaciones entre ellos. Para este análisis se consideraron únicamente los hongos diferentes a sus respectivos testigos, en evaluaciones distintas.

El Cuadro 3 muestra que ningún aislamiento de Cladosporium spp., resultó significativamente distinto $(p>0,05)$. Esto podría deberse a que no se contaba con los medios para identificar las especies, por lo que se podría haber trabajado con una o unas pocas especies. Además puede deducirse que indistintamente de donde fueron aisladas, tienen un mismo comportamiento como antagonista de Kuehneola spp.

De todos los ensayos realizados, en la Figura 4b se puede observar que Clados4 fue el único individuo que se puede separar estadísticamente del resto de hongos con diferencias significativas al testigo dentro de su evaluación. Del anterior análisis se excluyó a Cados4 ya que no cumplía con el supuesto de homogeneidad de varianza, esto porque su valor era 0 (Figura 4b). Sin embargo, se considera un aislamiento importante a considerar, pues inhibió totalmente la germinación de uredósporas de Kuehneola spp., como varios investigadores lo han demostrado en otras royas (Moricca et al.2001, Assante et al. 2004, Tsuneda y Hiratsuka 1979). 
Cuadro 3. Comparación de los hongos analizados como posibles antagonistas de Kuehneola spp., con diferencias estadísticas respecto a sus respectivos testigos. Los datos muestran la probabilidad.

\begin{tabular}{|c|c|c|c|c|c|c|c|}
\hline & Clados1 & Clados2 & Clados6 & Clados7 & Clados8 & Clados 9 & Clados 10 \\
\hline \multicolumn{8}{|l|}{ Clados1 } \\
\hline \multicolumn{8}{|l|}{ Clados2 } \\
\hline Clados6 & 0,5684 & 1,0000 & & & & & \\
\hline Clados7 & 0,8597 & 0,6426 & 0,6426 & & & & \\
\hline Clados8 & 0,2983 & 0,6837 & 0,6837 & & & & \\
\hline Clados9 & 0,8062 & 0,4150 & 0,4150 & 0,6261 & 0,1839 & & \\
\hline Clados10 & 0,6218 & 0,8679 & 0,8679 & 0,7170 & 0,5249 & & \\
\hline
\end{tabular}

\section{Evidencias microscópicas del antagonismo entre Cladosporium spp., y Kuehneola spp.}

En la Figura 5 se presentan algunas de las imágenes obtenidas cuando Cladosporium spp., interactuó con las uredósporas de Kuehneola spp., durante el proceso de germinación. En los conteos se logró observar grupos de uredósporas sin germinar rodeadas por hifas de Cladosporium spp. (Figura 5a). También hifas de Cladosporium spp., que estaban en contacto y en algunos casos
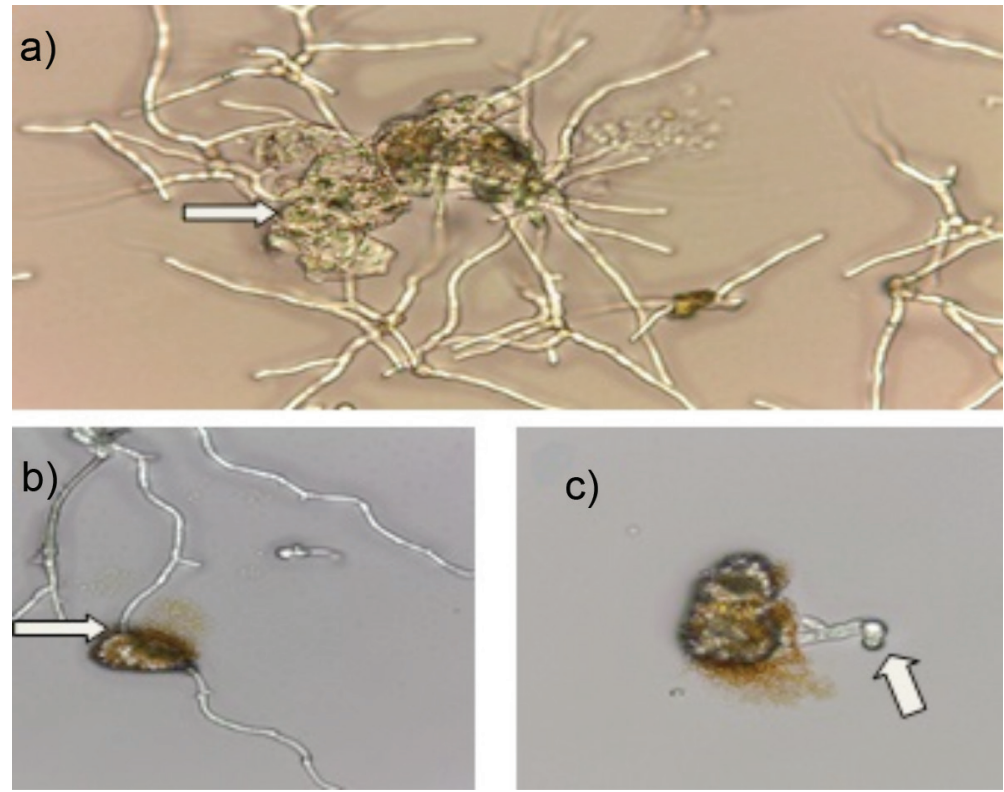

a) Grupo de uredósporas sin germinar rodeadas de hifas de Cladosporium spp., con la pared celular aparentemente dañada y sin el contenido celular normalmente visible.

b) Hifa de Cladosporium spp., atravesando una uredóspora.

c) Conidio de Cladosporium spp., que germina hacia la uredóspora y entra en contacto con ella.

Fig. 5. Uredósporas de Kuehneola spp., interactuando con conidios de Cladosporium spp. 
atravesaban las uredósporas (Figura $5 \mathrm{~b}$ y $5 \mathrm{c}$ ). De igual forma, fue común encontrar uredósporas con la pared celular aparentemente dañada y con lisis del contenido celular normalmente visible.

En estudios similares al realizado en esta oportunidad, Moricca et al. (2001) y Assante et al. (2004) observaron cómo Cladosporium tenuissimum hiperparasitaba las royas $C$. flaccidum (pino) y Uromyces appendiculatus (frijol). Dentro de las observaciones bajo el microscopio realizadas por estos autores, ellos destacaron grupos de esporas sin germinar en donde $C$. tenuissimum había crecido vigorosamente. También observaron cómo, al germinar los conidios de $C$. tenuissimum, algunos tendían a dirigirse hacia la espora de la roya y a adherirse a esta (en algunos casos) para penetrar y emerger por otro lado.

En otro estudio Tsuneda y Hiratsuka (1979) comprobaron que $C$. gallicola es un micoparásito altamente destructivo de la roya Endocronartium harknessii (pino). En este caso, el parasitismo ocurría tanto por el contacto de las hifas de $C$. gallicola con las esporas de la roya como por el contacto y penetración de estas. En muchos casos se observó que se destruía la membrana del propágulo de la roya, al estar en contacto con las hifas del parásito y eventualmente se desaparecía el citoplasma de la espora. Las observaciones visuales determinadas en este estudio, constituyen una evidencia más del efecto antagonista que el análisis estadístico mostró, indistintamente de la o las especies involucradas.

Al observar y realizar los conteos de las uredósporas de Kuehneola spp., se lograron apreciar ciertas similitudes con las observaciones de Moricca et al. (2001), Assante et al. (2004) y Tsuneda y Hiratsuka (1979) lo que constituye una evidencia de la asociación parasítica entre los distintos aislamientos de Cladosporium spp., sobre Kuehneola spp. La información obtenida en este trabajo, indica el potencial que tiene el género Cladosporium para usarse en el combate biológico de la roya de la mora y constituye el primer informe generado en Costa Rica de ese hongo como antagonista de Kuehneola spp., por lo que será necesario indagar más y realizar pruebas in vivo, pues no siempre la capacidad antagónica in vitro corresponde a un comportamiento igual en el campo (Cundom et al. 1999).

\section{LITERATURA CITADA}

Agrios, GN. 2005. Plant pathology. 5 ed. Florida, Estados Unidos. Elsevier academic press. 922 p.

Andrews, JH. 1992. Biological control in the phyllosphere. AR (Annual Review of Phytopathology) 30:603-635.

Arauz, LF. 2011. Fitopatología: un enfoque agroecológico. 2 ed. San José, Costa Rica. Universidad de Costa Rica. 519 p.

Assante, G; Maffi, D; Saracchi, M; Farina, G; Morrica, S; Ragazzi, A. 2004. Histological studies on the mycoparasitism of Cladosporium tenuissimum on uredinospores of Uromyces appendiculatus. Mycological Research 108(2):170-182.

Canjura, EM; Sánchez, V; Krauss, U; Samorriba, E. 2002. Reproducción masiva de Verticillium sp., hiperparásito de la roya del café, Hemileia vastatrix. Manejo Integrado de Plagas y Agroecología 66:13-19.

Castro, JJ; Cerdas, MM. 2005. Mora (Rubus spp.) cultivo y manejo poscosecha. San José, Costa Rica. MAG (Ministerio de Agricultura). 77 p.

Cundom, M; Mazza, S; Mazzanti, M; Gutierrez, S; Coutinho, M. 1999. Actividad antagónica in vitro de hongos saprófitos sobre Sclerotinia sclerotiorum. Corrientes, Argentina. UNNE (Universidad Nacional del Nordeste). 4 p.

Espinoza, J; Briceño, E. 2008. Canker and twig dieback of blueberry caused by Pestalotiopsis spp., and a Trucantella sp., in Chile. Plant Disease 92(10):14071414.

French, ER; Herbert, TT. 1980. Métodos de investigación fitopatológica. San José, Costa Rica. IICA (Instituto Interamericano de Cooperación para la Agricultura). $289 \mathrm{p}$.

Johnson, KB; Mahaffee, WF. 2010. Factors influencing epidemiology and management of blackberry rust in cultivated Rubus laciniatus. Plant Disease. 94(5):581-588.

Michel, A; Rebolledo, O; Lezama, R; Ochoa, M; Mesina, J; Samuels, G. 2001. Especies de Trichoderma en suelos cultivados con mango afectados por "escoba de bruja" y su potencial inhibitorio sobre Fusarium oxysporum y $F$. subglutinans. Revista Mexicana de Fitopatología 19(2):154-160.

Moricca, S; Ragazzi, A; Mitchelson, KR; Assante, G. 2001. Antagonism of two-needle pine stem rust fungi Cronartium flaccidum and Peridermium pini by Cladosporium tenuissimum in vitro and in planta. Phytopathology 91(5):457-468. 
Mouen, J; Njiayouom, I; Bieysse, D; Ndoumbè; Cilas, C; Nottéghem, L. 2008. Effect of shade on Arabica coffee berry disease development: toward an agroforestry system to reduce disease impact. Phytopahtology 98(12):1320-1325.

Orozco, M. 2003. Diagnóstico de las condiciones de manejo para implementar buenas prácticas agrícolas y buenas prácticas de manufactura en mora Vino orgánica, para la exportación y el mercado nacional. Tesis Lic. Cartago, Costa Rica. ITCR (Instituto Tecnológico de Costa Rica). 187 p.

Roiger, DJ; Jeffers, SN; Caldwell, RW. 1991. Occurrence of Trichoderma species in apple orchard and woodland soils. Soil Biology and Biochemistry 23(4):353-359.
Sharma, JK; Heather, WA. 1978. Parasitism of uredospores of Melampsora larici-populina Kleb. by Cladosporium spp. European Journal of Forest Pathology 8:48-54.

Tsuneda, A; Hiratsuka, Y. 1979. Mode of parasism of a mycoparasite, Cladosporium gallicola, on western gall rust, Endocronartium harknessii. Canadian Journal of Plant Pathology 1:31-36.

Yun, HY. 2010. Invasive fungi. Tropical american Kuehneola rust of Rubus-Kuehneola loeseneriana (en línea) Systematic Mycology and Microbiology Laboratory, Agricultural Research Service, Estados Unidos. Consultado 9 may. 2011. Disponible en http:// nt.ars-grin.gov/taxadescriptions/factsheets/index. cfm?thisapp $=$ Kuehneolaloeseneriana\&printtype $=$ 